\title{
Branching pattern formation that reflects the history of signal propagation
}

\author{
$\operatorname{AUTHOR}(\mathrm{S})$ :
}

Motoike, Ikuko; Takigawa-Imamura, Hisako

CITATION:

Motoike, Ikuko ...[et al]. Branching pattern formation that reflects the history of signal propagation. Physical Review E 2010, 82(4): 046205.

\section{ISSUE DATE:}

2010-10

URL:

http://hdl.handle.net/2433/134864

RIGHT:

(C) 2010 The American Physical Society 
PHYSICAL REVIEW E 82, 046205 (2010)

\title{
Branching pattern formation that reflects the history of signal propagation
}

\author{
Ikuko N. Motoike* \\ PRESTO, Japan Science and Technology Agency (JST), 4-1-8 Honcho Kawaguchi, Saitama 332-0012, Japan \\ and Institute for Integrated Cell-Material Sciences, Kyoto University, Yoshida Honmachi, Sakyo-ku, Kyoto 606-8501, Japan \\ Hisako Takigawa-Imamura \\ Institute for Integrated Cell-Material Sciences, Kyoto University, Yoshida Honmachi, Sakyo-ku, Kyoto 606-8501, Japan
}

(Received 15 March 2010; revised manuscript received 6 August 2010; published 5 October 2010)

\begin{abstract}
In living organisms, branching structures are often observed in open systems. During the process of structure formation/deformation, signal propagation can be observed. Branching paths often deform depending on the history of signal propagation. To gain a better understanding of the process of pattern formation that results in characteristic geometrical paths, we adopt a system in which the dynamics of path formation are correlated with signal propagation. This model involves both branch-generation dynamics and signal-propagation dynamics, and we introduced positive feedback between these two dynamic processes. We studied the geometrical properties of path deformation and the pattern of signal propagation using a discretized reaction-diffusion model. The proposed model can qualitatively reproduce different branching patterns and means of signal propagation. One remarkable result is that the mutual interaction of these two dynamic processes leads to autonomous wave generation, similar to a pacemaker or the generation of spiral waves. Because the autonomous wave generation in the signal is spontaneous, the shapes of the branching paths become distorted. We discuss the correlation between path deformation and signal propagation as a first step in understanding signal processing for such complex deformable paths.
\end{abstract}

DOI: 10.1103/PhysRevE.82.046205

PACS number(s): 05.45.-a, 05.10.-a, 87.19.1p

\section{INTRODUCTION}

There are many ordered complex patterns in nature, and such patterns can be observed in various fields of investigation. Living organisms form their own organs and even their entire bodies via autonomous formation processes with cross-talk between these organisms and external/internal signals. In the generation of these patterns, the formation of branching structures in open systems is often observed. For example, branching patterns can be observed with neurons, blood vessels, lungs, leaf veins, trees, bacterial colonies, protoplasmic streaming tubes of slime molds, ant trails, and so on. These branching structures have interesting features as effective transportation/propagation systems [1].

In general, the transport of materials or the propagation of signals is observed in these branching structures, and the branching structure itself can intrinsically affect transportation or propagation. A notable property of the transport of a substance is mass diffusion or flow (movement) conservation at a branch point. Branching tube morphology obeys this physical constraint [2-4]. When we consider the propagation of signals, it is important to realize that rather than materials, a "physical state" is moving. Thus, this movement can be faster and more robust than the flow or diffusion of material. For example, propagation of an action potential in the nervous system is a form of signal propagation. In this study, we focus on the interactions between branch formation and signal propagation.

To obtain a better understanding of the general features and functional aspects of branching pattern formation, it is

\footnotetext{
*i.n.motoike@icems.kyoto-u.ac.jp
}

important to determine how signal propagation or substance transport affects branching deformation. With regard to the effect of substance transport on path formation, Nakagaki $e t$ $a l$. recently reported a path-finding problem for a true slime mold that exhibited protoplasmic streaming tubes [5]. They proposed a simple algorithm for path formation between a sink and a single or multiple attractive sources [4]. The pattern of blood flow in the developing heart also plays a significant role in cardiac morphogenesis [6]. In contrast, typical patterns seen with nerve cells often depend on signal propagation $[7,8]$.

Regarding the neuron structure, recent technical advances have enabled the visualization and genetic manipulation of individual dendritic trees. These studies have led to the identification and characterization of molecules that are important for different aspects of dendrite development. Although much remains to be learned, the existing knowledge has allowed us to take initial steps toward obtaining a comprehensive understanding of how complex dendritic trees are built. If we focus on the functional aspects of signal processing within a branching structure, it would be useful to study the dynamics of branching pattern formation that reflect the history of signal propagation.

Several models that do not consider signal-processing dynamics have been proposed to describe complex branched dendritic pattern formation [2,3,9-13]. Bacterial colonies also exhibit typical diffusion-limited aggregation (DLA) patterns, and several models have been proposed to describe such pattern formation using reaction-diffusion systems $[10-12,14-16]$. We previously reported a simplified model for the formation of a branching pattern based on discrete reaction-diffusion dynamics [17].

In this study, we focused on the mutual interactions between branching pattern formation and signal propagation at 
the branches in a simplified model, and examined the spatiotemporal patterns that appeared.

\section{METHODOLOGY}

We propose a conceptual framework that includes a combination of dynamic processes with two different types of time constants. These dynamic processes, branching path formation and signal propagation, can be essentially described using a reaction-diffusion model. In general, a reactiondiffusion model can be described by a system of partial differential equations (PDEs). However, an enormous computational cost is required to solve these PDEs, especially if they include different time constants. To avoid this problem and clarify the interrelationships, we adopt coupled discrete dynamics, called cellular automata (CA). This includes a simplified branch formation model based on the author's previously proposed model [17] and wave-propagation dynamics with an excitable condition based on a model proposed by Gerhardt et al. [18,19].

There are no models for the formation of various branching patterns based on discrete RD systems, except for the author's proposed model; thus, in this paper, we adopt the author's model [17] to essentially describe branching pattern formation. This model is a simple three-variable CA system, which is a modification of an activator-depleted substrate system based on the RD concept for bacterial colony pattern formation [11]. This model can represent various branching patterns.

With regard to signaling wave-propagation dynamics, to represent the dynamics of wave propagation, generation, and annihilation, excitation pulse dynamics are adopted using a reaction-diffusion system comprising two variables. To simplify the description, the modified cellular automaton model of excitable media proposed by Gerhardt et al. is used $[18,19]$. This model represents the propagation dynamics of a real excitation wave well using a simple algorithm (e.g., curvature of wave and speed).

How these dynamic processes are coupled is outlined in the following. Signaling waves are allowed to exist only in the branching path. This effect is one manner of dynamic coupling. We add a positive feedback effect from wave propagation to branching pattern formation. We set that waves' propagation has the effect of propagating path elongation. Thus, increased wave propagation results in increased branching pattern growth. We describe each model component and its manner of coupling in detail in the next section.

In CA models, the target space is divided into discrete areas called cells. The evolution of the state of each cell with time is determined by simple intra- and intercell rules. A dynamic pattern or an entire structure can be generated from these local interaction rules. The spatial array of these cells is defined as a square, and the state of each cell within the array is updated in discrete time steps according to the present state of the cell and its neighbors. The inner state of each cell is described by a set of five variables: three for slow branching-path formation, "path maker" $(a)$, "growth factor" $(f)$, and "path trail" $(w)$, and two for fast wave propagation, "activator" $(u)$ and "inhibitor" $(v)$.
CA models have generally been anisotropic (primarily square or hexagonal), which has led to the problem that the anisotropy tends to be propagated into the resulting patterns. Here, we used Markus' method that involves a semirandom grid to prevent anisotropy of the diffusion effect [20]. A plane is divided into square cells and one point is placed randomly within each cell as the "cell center." We describe the center position of the $i_{\text {th }}-j_{\text {th }}$ cell by $(\hat{i}, \hat{j})$; for example, $i \leq \hat{i}<(i+1)$, where $\hat{i}$ and $\hat{j}$ are real numbers and $i$ and $j$ are integers. The random center positions of the cells are generated only once at the beginning of the simulation. A neighbor set of a cell $(i, j), N_{i j}$, is defined as all of the cells for which each cell center is within a circle of radius $R$ centered on the given cell $(i, j)$.

$$
N_{i j}^{x}=\left\{(k, l) \mid \sqrt{(\hat{k}-\hat{i})^{2}+(\hat{l}-\hat{j})^{2}} \leq R_{x}\right\},
$$

where $x$ corresponds to each variable. The circle radius corresponds to the diffusion coefficient, such that $R_{a}$ and $R_{f}$ correspond to $D_{a}$ and $D_{f}$, respectively. Hence, in the case where a variable assumes a binary value ( 0 or 1$)$, the number of cells for which the relevant variable is positive in the neighboring cell $(i, j)$ is

$$
E_{i j}^{x}=\#\left\{(k, l) \mid(k, l) \in N_{i j}^{x} \quad \text { and } \quad x_{k l}=1\right\} .
$$

\section{A. Branching-path formation}

The branching-path formation model adopted in this study is the author's previously proposed model, which was a reduced model based on a model of a bacterial colony formation proposed by Mimura et al. [11]: MSM model. The resulting patterns, particularly for the cases of most branching modes with the MSM model or with the author's previous model, are reminiscent of clusters arising from twodimensional DLA; these can be generated by a simple particle model for randomly branching patterns that grow via diffusion-limited processes [9]. However, the MSM model has a wider variety of patterns (i.e., it can represent not only DLA-like patterns, but also more dense patterns, disk-like patterns, and concentric ring patterns).

In the original MSM bacterial model, the states of the bacteria can be separated into two types. One type is active bacteria that move, grow, and undergo cell division, while another type is inactive bacteria that do nothing at all. An active bacterium exists at the edge of a colony and acts as a frontier for colony growth with the consumption of a nutrient. After nutrient consumption, if the nutrient becomes insufficient, an active bacterium converts to an inactive state. The nutrient diffuses throughout the medium.

In the MSM model, for which the system is described by PDEs, for the condition that the given amount of nutrient is small and the mobility of active bacteria is low (solid medium), nutrient diffusion dominates the pattern formation. Thus, it might be said that the field can be described as a Laplace field for the nutrient, and the pattern is regarded as DLA-like. In the parameter region, tip splitting and screening effects are observed. Because of the reduction of dynamics and discretization of the system, the field cannot be con- 
sidered to be Laplacian, although the author's previous model suggests it to be Laplacian [17].

In this study, the state of each cell can also be described by the three variables of "path maker" $(a)$, "growth factor" $(f)$, and "path trail" $(w)$. In the context of bacteria, these correspond to active bacteria, nutrient, and inactive bacteria, respectively. $a$ and $w$ assume binary values $\{0,1\}$, while $f$ is an integer $\left\{0,1, \ldots, f_{\max }\right\}$. The path variables are only permitted to assume on three cases: $a=1, w=0$ (path front in the cell), $a=0, w=1$ (path trail in the cell), and $a=0, w=0$ (no path in the cell).

When there are both a sufficient number of path makers in its neighborhood and sufficient growth factor in the cell $\left(f \geq f_{c}\right)$, the state of the path maker becomes $1(a \rightarrow 1)$. A "sufficient number" of path makers in the neighborhood is taken to be $a_{c}$. After $(a \rightarrow 1)$, when the number of path makers in the neighborhood is not larger than $a_{c}\left(E^{a} \leq a_{c}\right)$ or the growth factor in the cell itself is insufficient $\left(f<f_{c}\right)$, the state of the path maker becomes $0(a \rightarrow 0)$, and simultaneously, the state of the path trail becomes $1(w \rightarrow 1)$.

When a path maker exists in the cell $(a=1)$, it consumes the growth factor such that the growth-factor variable decreases $f_{\text {cons }}$ in time steps, where $f_{\text {cons }}$ is a positive integer. The growth factor continuously diffuses, and this is represented by averaging the growth factor in the neighborhood.

Therefore, the model for branching-path formation obeys the following rules, where distance between cell $(i, j)$ and cell $(k, l)$ is $r(k l-i j)=\sqrt{(\hat{k}-\hat{i})^{2}+(\hat{l}-\hat{j})^{2}}$.

$$
\begin{aligned}
& \text { (a1) if } f_{i j}(t) \geq f_{c} \text { and } E_{i j}^{a}(t)>a_{c} \\
& \Rightarrow a_{i j}(t+1)=1 \\
& \text { else if } a_{i j}(t)=1 \\
& \Rightarrow a_{i j}(t+1)=0, \quad w_{i j}(t+1)=1 \\
& \text { (a2) } f_{i j}(t+1)=\max \left[\frac{1}{\# N_{f r}} \sum_{r(k l-i j) \leq R_{f}} f_{k l}(t)-f_{\text {cons }} a_{i j}(t), 0\right] .
\end{aligned}
$$

Figure 1 shows a morphological map with the above rules. The pattern changed from a DLA-like pattern to a disk-like pattern, depending on the initial growth factor and the mobility of the path maker [17].

\section{B. Wave propagation}

In general, propagation of an excitation pulse with a reaction-diffusion system can be described with a pair of variables, "activator" $(u)$ and "inhibitor" $(v)$. An excitation pulse is generated when the input or stimulus exceeds the threshold. Initially, the activator increases autonomously, and the activator activates the inhibitor. Then, the inhibitor increases and inhibits the activator. Finally, the cell state returns to the resting state.

In Gerhardt's algorithm, the above dynamics are reduced to discrete rules. The excitation variable is permitted to have one of only two values, $u=0$ (unexcited) or $u=1$ (excited), whereas the recovery variable assumes integer values

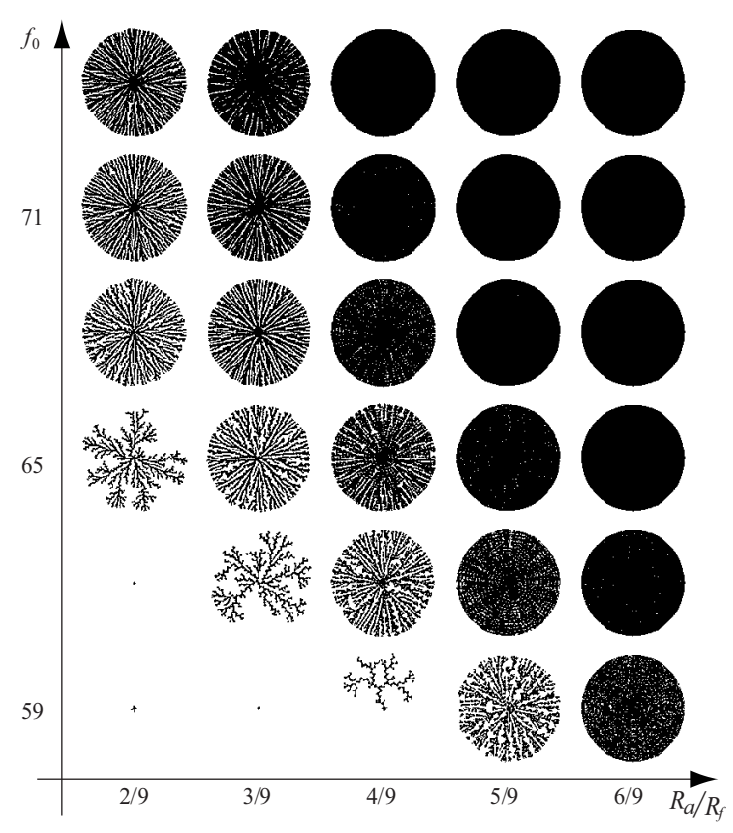

FIG. 1. Morphological map of the patterns observed for the algorithm without interactions. Black cells correspond to $w=1$ [17].

$\left(0,1, \ldots, V_{\max }\right)$. When $u=1$, the recovery variable $v$ increases until $v=V_{\max }$. When $v=V_{\max }, u$ switches to the unexcited state $(u=0)$ and $v$ decreases until $v=0$. The system is then at the stable rest state $(u=0, v=0)$.

Figure 2 shows a schematic diagram for excitable wave dynamics. To represent a spatially distributed excitable field, each cell obeys the dynamics in Fig. 2(b). The rule for the spatial spreading of an excitation wave is that an unexcited cell $(u=0)$ will become excited $(u=1)$ in the next time step if it is sufficiently recovered $\left(v \in\left[0, V_{e x c}\right]\right)$ and if a sufficient number of its "neighbors" are already in the excited state. In other words, the numbers of excited cells in the neighborhood of the cell $(i, j), E_{i j}^{u}$, needs to exceed a threshold, $k_{\text {exc }}(v)$. This should be an increasing function of $v$, because unexcited recovering cells that are farther from the resting state are more refractory and require more excited neighbors to push them to the excited state, $\left(E_{i j}^{u} \geq k_{\text {exc }}(v)\right)$. In Gerhardt's model, $k_{\text {exc }}(v)$ is written as

$$
k_{\text {exc }}(v)=k_{\text {exc }}^{0}+R_{u}\left(2 R_{u}+1\right)-k_{\text {exc }}^{0} \frac{v}{V_{\text {exc }}} .
$$
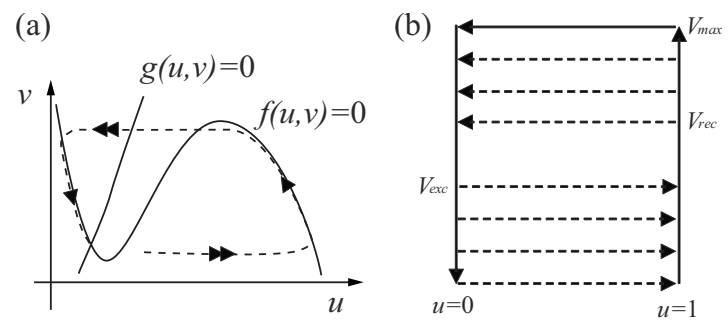

FIG. 2. (a) Typical phase-plane diagram for a differential equation model of an excitable medium. (b) Schematic of the state evolution of a cellular automaton [18]. 
TABLE I. Parameters set in this study.

\begin{tabular}{cccccccccccc}
\hline \hline$V_{\text {max }}$ & $V_{\text {exc }}$ & $V_{\text {rec }}$ & $g_{d w}$ & $g_{\text {up }}$ & $k_{\text {exc }}^{0}$ & $m_{u}$ & $f_{c}$ & $f_{\text {cons }}$ & $S_{c}$ & $a_{c}$ & $n$ \\
\hline 20 & 5 & 15 & 1 & 15 & 1 & 3 & 50 & 50 & 1 & 1 & 5 \\
\hline \hline
\end{tabular}

With regard to recovery from the excited state to the resting state, an excited cell $(u=1)$ will become unexcited $(u$ $=0$ ) in the next time step if $v \geq V_{\text {rec }}$. In Gerhardt's model, the recovery dynamics can be more complex, depending on the number of neighboring unexcited cells. In this study, to simplify the dynamics, an excited cell becomes unexcited independently.

The basic rules of wave propagation in this study obey the following:

$$
\begin{aligned}
& \text { (b1) if } u_{i j}(t)=0 \text { then } \\
& v_{i j}(t+1)=\max \left[v_{i j}(t)-g_{d w}, 0\right] \\
& \text { if }\left(v_{i j}(t) \leq V_{e x c} \text { and } E_{i j}^{u} \geq k_{e x c}\right) \\
& \Rightarrow u_{i j}(t+1)=1
\end{aligned}
$$

(b2) if $u_{i j}(t)=1$ then

$$
\begin{aligned}
& v_{i j}(t+1)=\min \left[v_{i j}(t)+g_{u p}, V_{\max }\right] \\
& \text { if }\left[v_{i j}(t) \geq V_{r e c}\right] \\
& \Rightarrow u_{i j}(t+1)=0
\end{aligned}
$$

where $g_{u p}$ and $g_{d w}$ are positive integers.

\section{Interaction feedback}

Here we introduce interaction feedback between branching-formation dynamics and wave-propagation dynamics. In the coupled system, waves are allowed to exist only in the branching path (cells in which a path trail or path maker is positive). In this study, we assumed a simple interaction: positive feedback between the coupled dynamic processes. Thus, branches at which a wave propagates grow or extend, while those without wave propagation shrink. Therefore, a sufficient history of wave propagation at a branch is necessary in order to extend the branch. In contrast, to investigate branch deformation, to include the case when a resting path trail changes to a path maker, the requirement for becoming a path maker can now be satisfied by either a sufficient number of path makers in its neighborhood or the presence of a path trail.

The CA model obeys the rules given below. At each step, wave-propagation dynamics are calculated by the following. These rules are applied only to the cells where the path factor (path maker or path trail) is positive. The difference from stand-alone wave-propagation rules (b1) and (b2) is the existence rule (3). Rule (3) represents the integration of the history of wave propagation $\left(S_{i j}^{u}\right)$, in the neighborhood of the target cell and constant decay to zero.
(1) if $u_{i j}(t)=0$ then

$$
\begin{aligned}
& v_{i j}(t+1)=\max \left[v_{i j}(t)-g_{d w}, 0\right] \\
& \text { if }\left[v_{i j}(t) \leq V_{e x c} \text { and } E_{i j}^{u} \geq k_{e x c}\right] \\
& \Rightarrow u_{i j}(t+1)=1
\end{aligned}
$$

(2) $u_{i j}(t)=1$ then

$$
\begin{aligned}
& v_{i j}(t+1)=\min \left[v_{i j}(t)+g_{\text {up }}, V_{\text {max }}\right] \\
& \text { if } \quad\left(v_{i j}(t) \geq V_{\text {rec }}\right) \\
& \text { then } \Rightarrow u_{i j}(t+1)=0
\end{aligned}
$$

(3) $S_{i j}^{u}(t+1)=S_{i j}^{u}(t)+m_{u} E_{i j}^{u}(t)-1$

To implement the difference in time constants, the following branching rules run every $n$th time step in the signalpropagating algorithm $\left(\tau_{(\text {branch })} / \tau_{(\text {signal })}=n\right)$.

(4) if $f_{i j}(t) \geq f_{c}$ and $S_{i j}^{u}(t) \geq S_{c}$ and

$$
\begin{gathered}
{\left[E_{i j}^{a}(t)>a_{c} \text { or } w_{i j}(t)>0\right]} \\
\Rightarrow a_{i j}(t+1)=1, \quad S_{i j}^{u}(t+1)=0
\end{gathered}
$$$$
\text { else if } a_{i j}(t)=1
$$

$$
\Rightarrow a_{i j}(t+1)=0, w_{i j}(t+1)=1
$$

(5) $f_{i j}(t+1)=\max \left[\frac{1}{\# N_{f r(k l-i j)}} \sum_{R_{f}} f_{k l}(t)-f_{\text {cons }} a_{i j}(t), 0\right]$.

(6) if $w_{i j}(t)>0$ and $S_{i j}^{u}(t) \leq S_{c}$ and $E_{i j}^{w}(t)<w_{c}$

$$
\Rightarrow w_{i j}(t+1)=0 \text {. }
$$

One difference from the stand-alone branching path formation rules (a1)-(a2) is the addition of the necessary condition in rule (4); the history of wave propagation that is counted by rule (3) and the permission of restitution of a path maker from a path trail. To maintain an existing path, integration of the history state of wave propagation in a cell $\left(S^{u}\right)$ needs to be larger than a critical value of $S_{c}$. The cell shrinks if the integration variable $S^{u}$ is not larger than $S_{c}\left(S^{u} \leq S_{c}\right)$, and if there is insufficient $E^{w}\left(E^{w}<w_{c}\right)$, the inner state becomes $w$ $=0$ with probability $p=0.1$ [rule (6)]. This rule reflects degeneration of the path, so that the path decreases in the absence of signal propagation, and the edges tend to shrink more easily than the center of the path. 


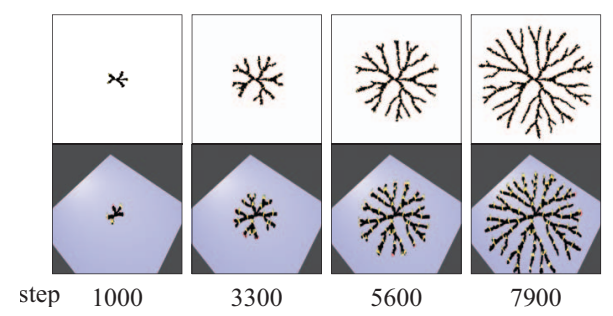

FIG. 3. (Color) Time-evolution of non dense pattern formation at time steps 1000, 3300, 5600, and 7900. The parameters are $R_{a}$ $=2$ and $f_{0}=68$. In the top row, the black, yellow, and red cells correspond to $w=1, u=1$, and $a=1$, respectively. The bottom row shows corresponding profiles of the excitation wave $u$ in a quasithree-dimensional representation. The dark region corresponds to $w=1$.

\section{RESULTS}

This CA model has three major parameters: the initial value of the growth factor $f_{0}$, and $R_{a}$ and $R_{f}$, which correspond to the diffusion coefficients of the path maker and the growth factor, respectively. We calculated the rules for a square lattice of lateral size $L=500$. The system was initially prepared by assigning each cell to a position $(\hat{i}, \hat{j})$ $=\left(i+\eta_{i j}^{i}, j+\eta_{i j}^{j}\right)$, with $\eta$ assuming different, uncorrelated random numerical values. These random values were assumed to be uniformly distributed in the interval $(0,1)$, where the unit cell length $d=1$. The growth factor $f(0)=f_{0}$ was assumed to be uniformly distributed and the path maker $u(0)$, path trail $w(0)$, activator $u(0)$, and inhibitor $v(0)$ were given values of zero, except for within a small central circle where $a(0)=u(0)=1$. Periodic stimuli $(u=1)$ were input to the small central circle every 25 time steps. In this paper, we used the parameters $R_{f}=9, R_{u}=2$, and $w_{c}=7$, and other values were set as in Table I.

Figure 3 and Fig. 4 [21] show the results of the patternformation process obtained for different values of $R_{a}$ and $f_{0}$. The magnitude of $R_{a}$ indicates the mobility of the path maker $a$ in the field when $R_{f}$ is constant. Therefore, a larger $R_{a}$ means that it is easier to move farther to take up the growth factor. Under these conditions, because of an insufficient amount of growth factor, the path maker $a$ tries to take up the limited growth factor (Figs. 3 and 4). As a result, the cluster of path makers becomes divided into several clusters, and a branching pattern appears as path trails. In this system, the propagation of excitation waves is needed in order to elongate the paths. The results in Figs. 3 and 4 show propagation

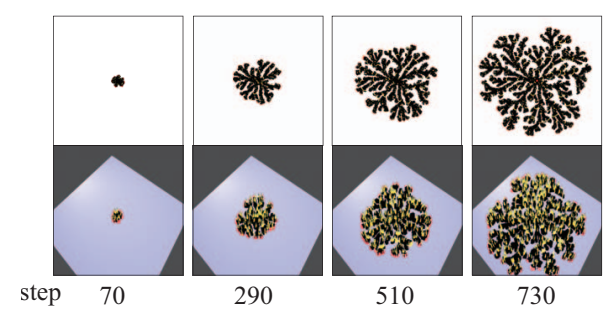

FIG. 4. (Color) Time evolution of dense pattern formation at time steps 70, 290, 510, and 730. The parameters are $R_{a}=5$ and $f_{0}=77$.

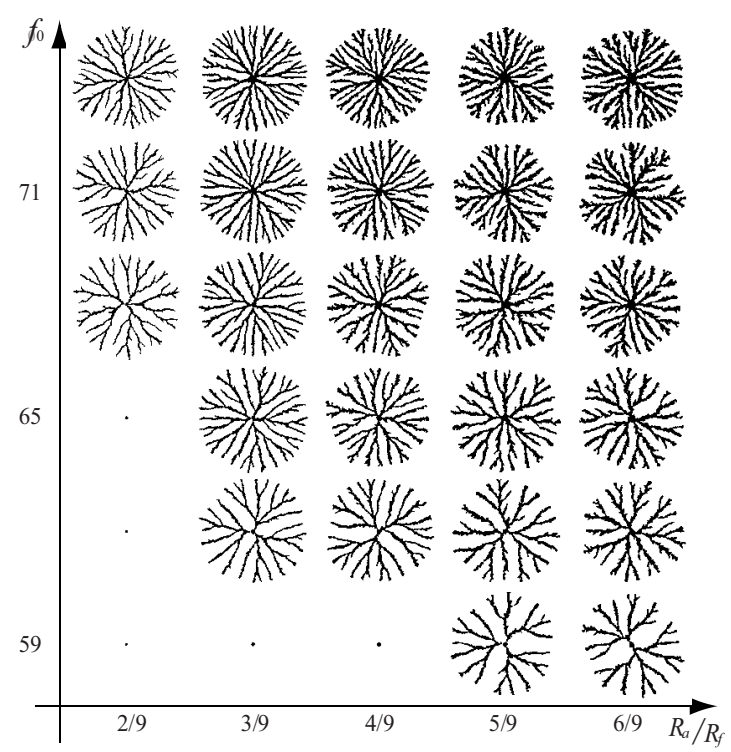

FIG. 5. Morphological map of the patterns observed in the algorithm with interaction. The region of the initial growth-factor value is the same as that in Fig. 1. Black cells correspond to $w=1$.

waves and path elongation. Path makers are seen at the ends of paths. In Fig. 3, the propagation of waves is sparse, concentric, and dominated by periodic input from the center area (see [21]).

However, in Fig. 4, despite of the same input scheme, propagating waves appear to be more dense and disordered. During the time series of pattern formation and wave propagation in Fig. 4, desultory generation of waves out of a center is seen, where an external input is not given. In other words, waves occur spontaneously. Because of this behavior of wave propagation, the pattern becomes thicker (see [21]).

To provide an overview of how the pattern features depend on the parameters, for comparison to the morphological map in Fig. 1, Fig. 5 shows a morphological map with coupled dynamics using rules (1)-(6). The parameters used for branch formation are the same as those shown in Fig. 1, which shows noncoupled dynamics. The parameter region within which a branching pattern is observed is larger than that with noncoupled dynamics, and the nature of branching becomes straighter and less dense. In this paper, the conditions are set so that pattern growth arises from a center seed and external input signals are constantly provided; thus, a shrinking effect does not appear.

Figure 6 shows a morphological map with the same conditions as in Fig. 5, except that the initial value of the growth factor $f_{0}$ is higher. The resulting patterns appear to quantitatively show two different types of patterns. Especially in the region with higher growth factor for the condition $R_{a}=5$, the patterns bend more and are anisotropic. In this region, propagating pulses interact so that the path region grows, and a rotating wave occurs to make a spiral wave. Thus, from that location, the path grows automatically with the autonomous generation of pulses, as in a pacemaker. As a result, the growth rate increases dramatically and the branches become thick.

To quantitatively describe the features of these patterns in a two-dimensional setting, we analyzed the fractal nature, the 


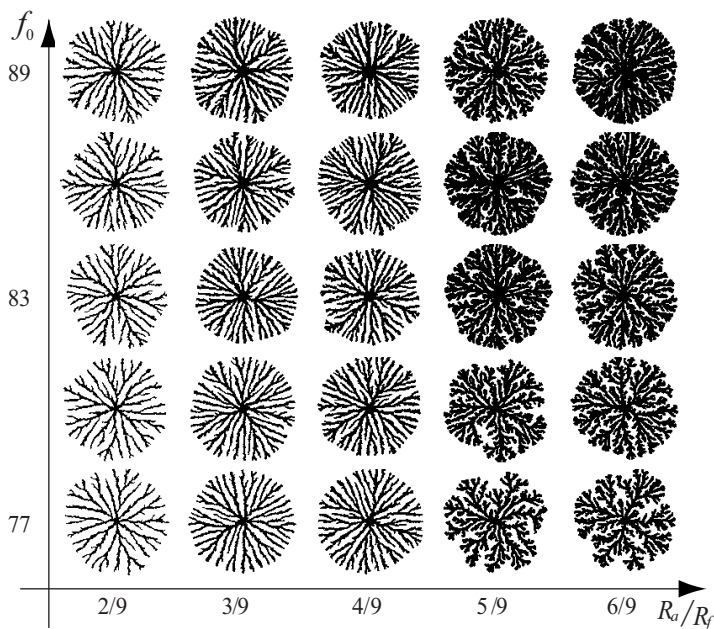

FIG. 6. Morphological map of the patterns observed in the algorithm that included interaction. The region shown for the initial growth-factor value is higher than that in Fig. 1. Black cells correspond to $w=1$.

number of branches, and the growth rate of the patterns obtained with the current CA model. The fractal dimension was calculated using the radius-of-gyration method [22]. A log$\log$ plot is shown in Fig. 7. The vertical axis is the number of cells in which path trails $w$ are positive and the horizontal axis is the pattern's radius of gyration in which the unit of length is the cell length. The slopes of the plots represent the fractal dimensions.

The details of the patterns changed depending on the random number used to determine the cell centers. Figure 8-10 show representative data for an average of 50 samples of the fractal dimensions, the number of branches, and the growth rate, respectively. The fractal dimension and the number of branches were calculated for the patterns at the moment when patterns grew to a given size: the farthest edge of branches arose at a distance of 230 unit cells from the center. The growth rate was calculated as follows. The distance from the center of a pattern to the outermost edge increased nearly monotonically with growth. The growth rate depended on the existence of spiral wave generation. Thus, the growth rate

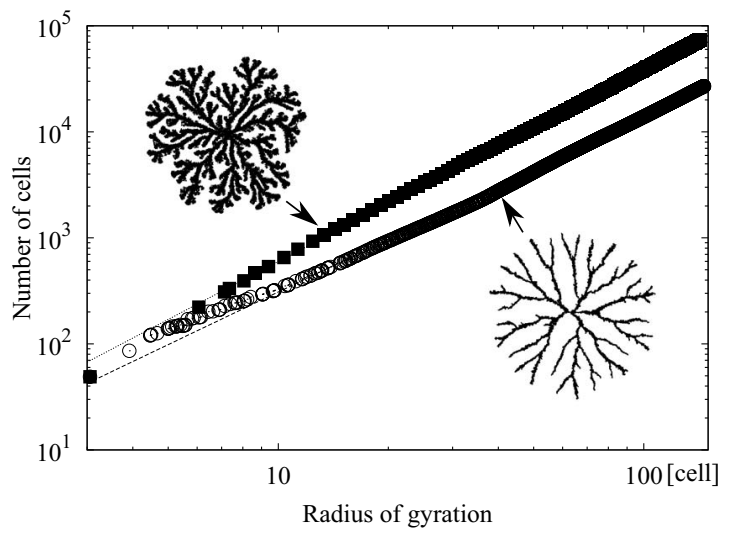

FIG. 7. Radius of gyration method for $R_{f}=9,(\bigcirc): R_{a}=2, f_{0}$ $=68$, and $(\mathbb{\square}): R_{a}=5, f_{0}=77$. The fractal dimensions are 1.65 and 1.81 , respectively.

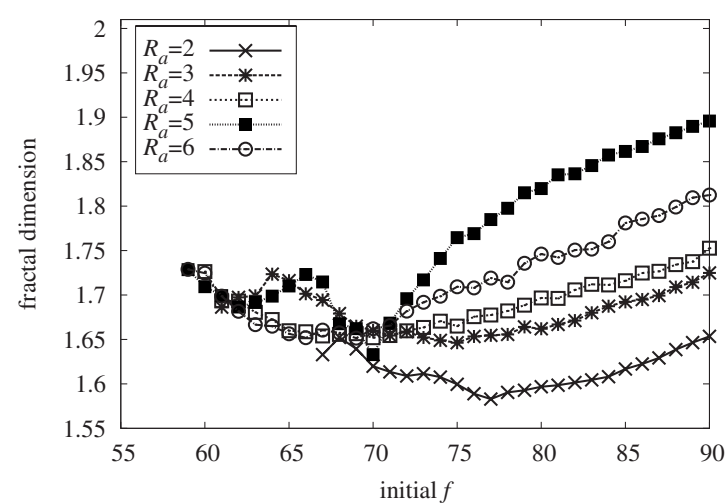

FIG. 8. Fractal dimensions of patterns observed with changes in the parameters $f_{0}$ and $R_{a}$.

was calculated in terms of the growth rate of the outermost edge of the pattern after the pattern radius was larger than 180.

The well-branched pattern seen with the DLA model is a fractal pattern with a fractal dimension of 1.715 [23]. With noncoupled dynamics, as shown in Fig. 1 , when $f_{0}$ and $R_{a}$ increase, the pattern becomes denser, and the fractal dimension approaches 2.0 [17]. In contrast, with coupled dynamics, patterns continue to branch with increasing $f_{0}$, and the fractal dimension gradually approaches 2.0 for $f_{0}>70$ (Fig. 8 ). In the current model, patterns for $R_{a}=5$ and $R_{a}=6$ show larger fractal dimensions for $f_{0}>70$.

In Fig. 9, the number of branches for $R_{a}=5$ shows a trend different from other $R_{a}$ values, where the rate of increase changes around $f_{0}=70$, and the rate of increase for $f_{0}>70$ is different from that for $f_{0}<70$ and other values of $R_{a}$.

In Fig. 10, for $R_{a}=5$, the graph increases dramatically around $f_{0}=70$, and for $f_{0}>70$, the growth rate maintains a higher value than for other values of $R_{a}$. These results suggest that patterns may change qualitatively around $f_{0}=70$ for $R_{a}=5$.

\section{DISCUSSION}

We calculated the fractal dimensions, the numbers of branches, and the growth rates for these patterns. These values represent the two-dimensional features of the patterns that result in signal propagation. Especially for $R_{a}=5$ and regions with a high $f_{0}$ value, patterns have larger fractal dimensions, more branches, and these branches appear to drift more and are thicker. For this parameter region, during the process to make patterns that drift more and are thicker, autonomous wave propagation is observed at multiple sites. A typical example of this kind of behavior is shown in reference [21].

With regard to the mechanisms of wave generation, this kind of behavior is often generated at the growth point of each path. By enlarging the point of wave generation, Fig. 11 shows a typical mechanism for wave generation. The scenario for autonomous wave generation is the following. If the excitable path area is fixed, an excitation wave propagates to the end point of the path and cannot reverse its incoming direction because of the presence of a refractory area 


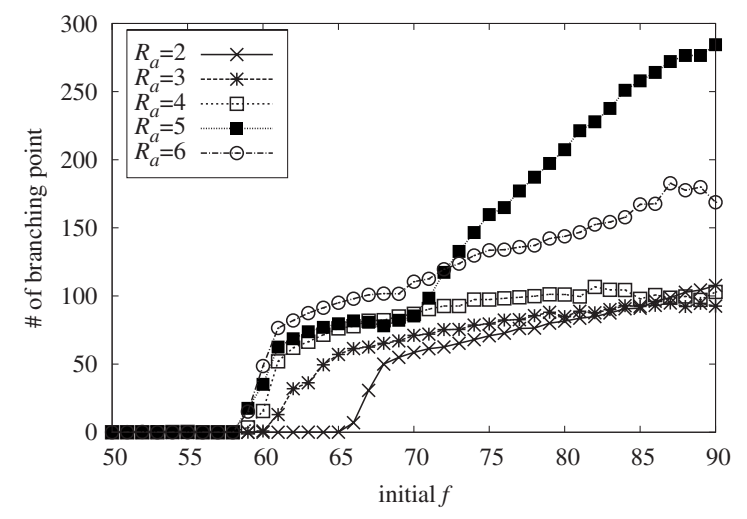

FIG. 9. Numbers of pattern branches observed with changes in the parameters $f_{0}$ and $R_{a}$.

behind propagating excitation wave. However, when the path extends around the tip where the wave arrives, a new excitable neutral area can appear at the front of the tip (red region in Fig. 11, panel 2). If so, the wave propagates to the new neutral area (yellow region in Fig. 11, panel 2), and then propagates along both sides of the path and returns in the in-coming direction (yellow regions in Fig. 11, panels 3, 4, and 5). After they return back to both sides of the original path, waves can penetrate into the original path because of completion of the path's refractory state (black regions in Fig. 11). Then, a couple of waves collide in the path (yellow region in Fig. 11, panel 7) and an out-going wave generates a new path (red region in Fig. 11, panel 8).

In this way, recursive wave propagation and generation occur. Once a pair of rotating waves develops, rotation is maintained. This manner of wave propagation can be said to be a kind of spiral wave. Because of this continuing recursive wave propagation, it can be considered that a path becomes thicker because of the additional side region of the path with a returning wave. At the thicker end point, tipsplitting can occur more readily because of the competition for obtaining the growth factor or to the instability of the interface. Also, the branch tip grows faster with a frequent recursive wave than with a sparse wave from the center. In this way, the pattern tends to be thicker, has more branches, and more drifting is apparent with frequent branching.

In the morphological maps, Figs. 5 and 6, successive periodic inputs are given at the center of the pattern. However,

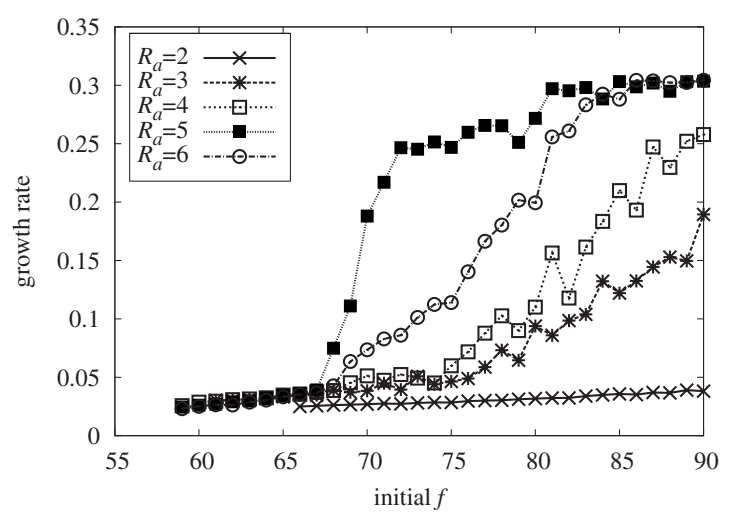

FIG. 10. Growth rates of the patterns observed with changes in the parameters $f_{0}$ and $R_{a}$.

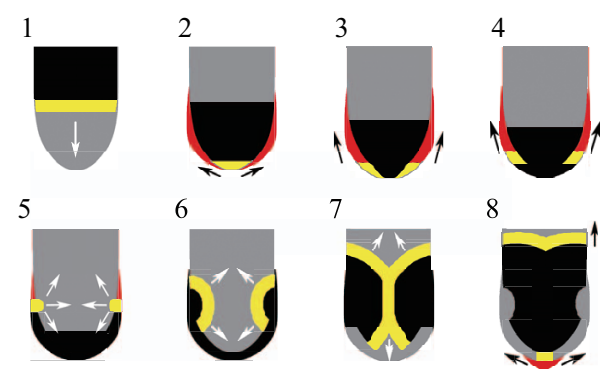

FIG. 11. (Color) Frame format for the process of spiral wave generation at the end of a path. Yellow and black regions correspond to the wave front (excited region) and wave back (unexcitable refractory region), respectively. Gray and red regions correspond to the existing and newly formed neutral branching paths, respectively.

once a spiral wave is generated, a pattern begins to grow autonomously and independently of any external input. Thus, if external inputs stop after several time steps, the pattern cannot continue to grow without internal spiral waves. To obtain this feature, we changed the input conditions from those in the previously mentioned scheme. We made the external input stop after 10 iterations. Figure 12 shows a morphological map to clarify the parameter region where spiral waves are generated.

Figure 13 shows the probability of formation of a branching pattern with the first transient external inputs, which represents the occurrence of spiral wave generation. The details of the patterns change depending on the random number used to determine the cell centers. This figure shows representative data for an average of 50 samples.

In particular, when $R_{a}=5$, it appears that the spatiotemporal conditions of wave propagation and path elongation are suitable to generate a spiral wave. The difference in the time constant between coupled dynamics, in other words, the competition between the wave-propagation rate, including a

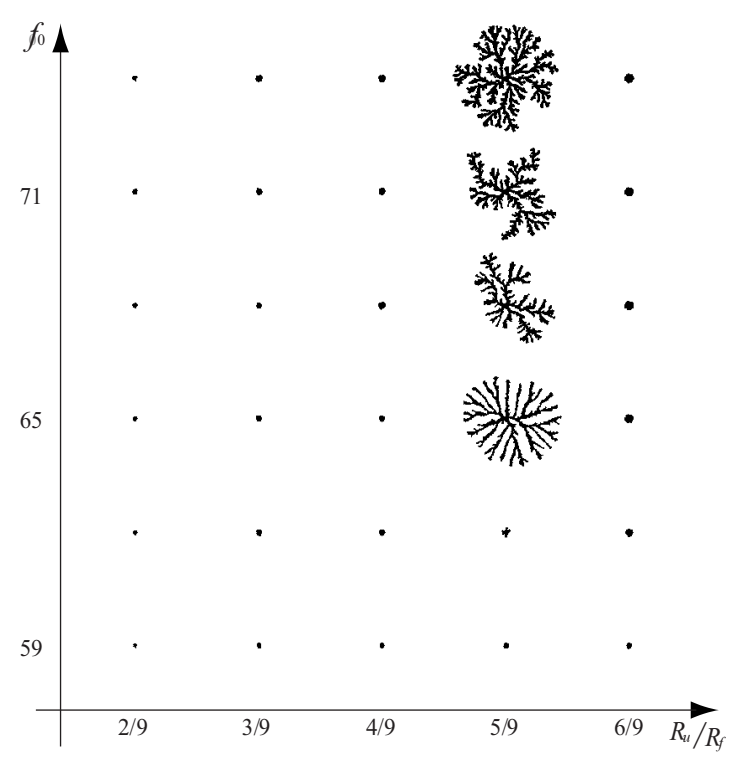

FIG. 12. Morphological map of the patterns observed with the algorithm that includes interaction, with the first transient external inputs. Black cells correspond to $w=1$. 


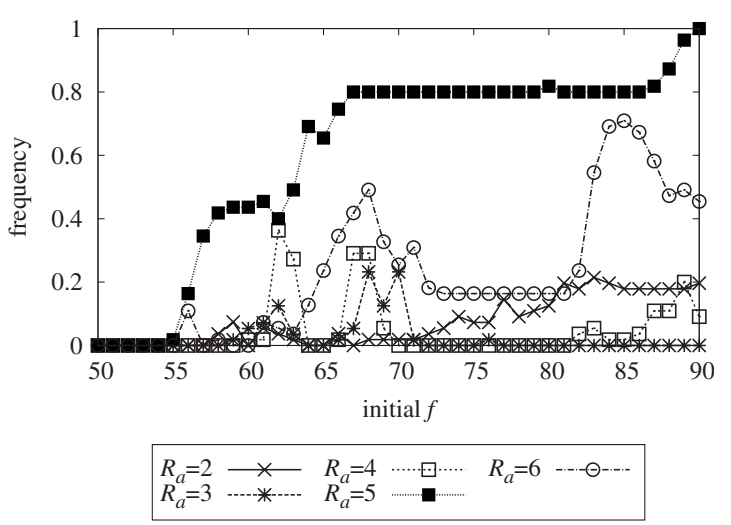

FIG. 13. Probability of formation of branching patterns observed with changes in the parameters $f_{0}$ and $R_{a}$.

nonexcitable period and the path elongation rate, may be essential for generating spiral waves. For the condition $R_{a}$ $=5$, the wavelength, refractory period, and timing for the formation of a new path may be in balance.

However, a theoretical analysis may not be applicable because the current model is described using discrete dynamics. CA has reduced discrete dynamics; thus, because of this simplicity, it is difficult to describe the intricate balancing conditions. In contrast, continuous PDE systems can have more degrees of freedom by choosing the form of the functions and the relationships between variables. Thus, there may exist a wider range of the parameter area in which recursive waves are generated with a continuous PDE system, in which branching path formation is coupled with excitation wave propagation. It would be interesting to study if this recursive process occurs with a PDE system.

Branching patterns are often observed in physical and living systems. A typical example in a physical system is crystal growth or viscous fingering, in which dendritic growth is driven by the instabilities of a pattern's interface [24]. For a living system, a bacterial colony shows branching patterns under the conditions of a solid culture medium and low nutrient levels. For these phenomena, the field (concentration field, pressure field, etc.) can be regarded as a Laplace field, and tip-splitting effects are driven by interface instabilities. Correspondingly with the proposed model, outward signal propagation enhances the effects of this instability as follows: tips tend to grow, and a longer branch induces a screening effect for growth of other branches in its neighborhood.

Instead, in this study, we considered wave propagation as a "signal." Thus, we will design our future studies of this model in relationship to biological systems. However, the model proposed here is rather simple; thus, it will not be easy to find straightforward relationships between this model and a biological process. To link this model with biological systems, possibilities are systems of nerves, lungs, blood vessels, slime molds, and others. Particularly for systems of nerves, signal propagation and deformation of branching paths is quite an attractive issue.

In the nervous system, branching patterns appear in many shapes and sizes [25]. In recent biological neural research, it has become obvious that the spatial geometry of individual neurons is crucial for neural computation [26]. Single neurons can distinguish among image patterns because of their geometrical properties [27]. Feinerman et al. reported that mesoscale logical devices could be created in vitro based on the geometrical design of neuron cultures [28].

Regarding other aspects in the context of the functionality of characteristic path geometry, one possibility for a relationship is an efficiency strategy for signal propagation against resources for path formation. In other words, in these biological phenomena there are certain essential features, such as some kind of conserved quantity (i.e., air or blood) at the branching point or protoplasm in the tube of a slime mold.

One major difficulty for investigating biological systems is the presence of significant factors in relation to a targeted phenomenon. For example, in order to study the relationship between signal propagation and deformation of cell's shape in the nervous system, we should take into account calcium wave propagation, spatial distributions of ion channels, hierarchical structures, and networks of many types of proteins and other molecules. In contrast to this, the proposed model is rather simple and there are several possible extensions, such as the introduction of a conserved quality for a path resource, connecting multiple branching patterns and input signals, and so on. A simple physical model, such as the proposed model, may possibly allow fundamental studies of a signal-processing system in which functions can change autonomously, as in living systems.

\section{CONCLUSION}

We adopted a system in which the dynamics of path formation were correlated with signal propagation. This model involved both branch-generation dynamics and signalpropagation dynamics, and the interaction between these two dynamic processes was set to have positive feedback. The proposed model used five variables: path makers, path trails, and growth factors to represent branching-path formation, and activators and inhibitors to represent signal propagation. The essential feature of branching pattern formation is competition to obtain a growth factor.

An important feature of this model is the mutual interaction between dynamic processes with different time constants. This mutual interaction leads to autonomous wave generation, similar to a pacemaker or the generation of spiral waves. Because of the spontaneous occurrence of autonomous wave generation in the signal, the shapes of the branching paths become distorted. Qualitative differences of the patterns are important to study the mechanisms of pattern generation and the functions of the target pattern.

In this paper, no additional growth factor was supplied after the given initial amount. Under a constant supply of growth factor, various patterns appear. For example, branch growth, both outward and inward, may develop. This effect may be clearer in a three-dimensional system because there are more degrees of freedom for growing direction than twodimensional system. Hence, branching form may be observed clearer in the three-dimensional system due to a lower probability of overlap of paths compared to two-dimensional 
system. The effects of a constant-feeding system will be addressed in a future study.

Several studies [29-38] have provided interesting examples of information-processing using suitably simple geometries for RD fields based on the concept of field computation using time-sequential information. One of the next questions to be addressed is how signal processing can be driven by the properties of a complex geometry, such as a well-branched pattern. Numerical simulation and simple experiments are effective for identifying the effects of a complex geometry on signal processing.

These interesting properties of field computation can actually be realized with a RD system, such as the BZ chemical reaction [39]. However, a problem with the $\mathrm{BZ}$ reaction is its fragility, as a liquid reaction medium is not suitable for use as a practical computing machine. A remaining problem is how to obtain a conventional excitable field on a reliable solid circuit to construct a field computer. Thus, these operations together with time-sequential information could be implemented into a solid circuit using modern electronic technology, as with semiconductor production [40] or on a solid surface [41].

A simple algorithm to describe various branching geometries may also be a good tool for simulating and realizing a solid circuit. The main advantage of the method presented here over PDE calculations is its simplicity. As an extension of the present study, it may be useful to determine whether a network of branching devices can perform signal processing, and to identify the actual mechanism of the time operation in a real biological system. Therefore, further studies are needed.

\section{ACKNOWLEDGMENTS}

We are grateful to Professor Kenichi Yoshikawa and Professor Konstantin Agladze for their kind discussions and comments. This research was supported by JST PRESTO program and by JSPS KAKENHI (Grant-in-Aid for Young Scientists (B), Grant No. 20700213), Japan.
[1] E. Scott and L. Luo, Nat. Neurosci. 4, 359 (2001).

[2] T. Miura, Curr. Top Dev. Biol. 81, 291 (2008).

[3] P. Carmeliet and M. Tessier-Lavigne, Nature (London) 436, 193 (2005).

[4] A. Tero, R. Kobayashi, and T. Nakagaki, J. Theor. Biol. 244, 553 (2007).

[5] T. Nakagaki, H. Yamada, and A. Toth, Nature (London) 407, 470 (2000).

[6] J. R. Hove, R. W. Koster, A. S. Forouhar, G. Acevedo-Bolton, S. E. Fraser, and M. Gharib, Nature (London) 421, 172 (2003).

[7] J. Y. Hua, M. C. Smear, H. Baier, and S. J. Smith, Nature (London) 434, 1022 (2005).

[8] D. L. Dickstein, D. Kabaso, A. B. Rocher, J. I. Luebke, S. L. Wearne, and P. R. Hof, Aging Cell 6, 275 (2007).

[9] T. A. Witten and L. M. Sander, Phys. Rev. Lett. 47, 1400 (1981).

[10] A. M. Lacasta, I. R. Cantalapiedra, C. E. Auguet, A. Peñaranda, and L. Ramírez-Piscina, Phys. Rev. E 59, 7036 (1999).

[11] M. Mimura, H. Sakaguchi, and M. Matsushita, Physica A 282, 283 (2000).

[12] H. Levine and E. Ben-Jacob, Phys. Biol. 1, 14 (2004).

[13] K. Sugimura, K. Shimono, T. Uemura, and A. Mochizuki, PLOS Comput. Biol. 3, e212 (2007).

[14] E. Ben-Jacob, O. Schochet, A. Tenenbaum, I. Cohen, A. Czirok, and T. Vicsek, Nature (London) 368, 46 (1994).

[15] K. Kawasaki, A. Mochizuki, M. Matsushita, T. Umeda, and N. Shigesada, J. Theor. Biol. 188, 177 (1997).

[16] S. Kitsunezaki, J. Phys. Soc. Jpn. 66, 1544 (1997).

[17] I. Motoike, J. Phys. Soc. Jpn. 76, 034002 (2007).

[18] M. Gerhardt, H. Schuster, and J. J. Tyson, Physica D 46, 392 (1990).

[19] M. Gerhardt, H. Schuster, and J. Tyson, Science 247, 1563 (1990).
[20] M. Markus and B. Hess, Nature (London) 347, 56 (1990).

[21] See supplementary material at http://link.aps.org/supplemental/ 10.1103/PhysRevE.82.046205, Movie1.mpg for a movie of growing process which corresponds to Fig. 3 and Movie2.mpg for a movie of growing process which corresponds to Fig. 4.

[22] P. Meakin, Phys. Rev. A 27, 604 (1983).

[23] S. Tolman and P. Meakin, Phys. Rev. A 40, 428 (1989).

[24] Branching in Nature: Dynamics and Morphogenesis of Branching Structures, from Cell to River Networks (Centre de Physique des Houches), 1st ed., edited by V. Fleury, J.-F. Gouyet, and M. Leonetti (Springer, New York, 2001).

[25] G. Stuart, N. Spruston, and M. Häusser, Dendrites (Oxford University Press, USA, 2007).

[26] H. Agmon-Snir, C. E. Carr, and J. Rinzel, Nature (London) 393, 268 (1998).

[27] B. W. Mel, Neural Comput. 4, 502 (1992).

[28] O. Feinerman, A. Rotem, and E. Moses, Nat. Phys. 4, 967 (2008).

[29] K. Agladze, R. R. Aliev, T. Yamaguchi, and K. Yoshikawa, J. Phys. Chem. 100, 13895 (1996).

[30] O. Steinbock, P. Kettunen, and K. Showalter, J. Phys. Chem. 100, 18970 (1996).

[31] I. Motoike and K. Yoshikawa, Phys. Rev. E 59, 5354 (1999).

[32] I. N. Motoike, K. Yoshikawa, Y. Iguchi, and S. Nakata, Phys. Rev. E 63, 036220 (2001).

[33] I. N. Motoike and A. Adamatzky, Chaos, Solitons Fractals 24, 107 (2005).

[34] T. Ichino, Y. Igarashi, I. N. Motoike, and K. Yoshikawa, J. Chem. Phys. 118, 8185 (2003).

[35] H. Nagahara, T. Ichino, and K. Yoshikawa, Phys. Rev. E 70, 036221 (2004).

[36] J. Gorecka and J. Gorecki, Phys. Rev. E 67, 067203 (2003).

[37] J. N. Gorecka, J. Gorecki, and Y. Igarashi, J. Phys. Chem. A 111, 885 (2007).

[38] K. Yoshikawa, I. N. Motoike, T. Ichino, T. Yamaguchi, Y. Iga- 
rashi, J. Górecki, and J. N. Górecka, Int. J. Unconv. Comput. 5, 3 (2009).

[39] A. Adamatzky, B. De Lacy Costello, and T. Asai, Reaction Diffusion Computers (Elsevier, New York, 2005).

[40] M. Takahashi, T. Asai, T. Hirose, and Y. Amemiya, Int. J.
Bifurcation Chaos Appl. Sci. Eng. 17, 1713 (2007).

[41] N. A. Vlasenko, H.-G. Purwins, Z. L. Denisova, Ya. F. Kononets, F.-J. Niedernostheide, L. I. Veligura, and S. Zuccaro, Semicond. Phys., Quantum Electron. Optoelectron. 7, 82 (2004). 